\title{
Using the Vapor Pressure of Pure Volatile Organic Compounds to Predict the Enthalpy of Vaporization and Computing the Entropy of Vaporization
}

\author{
Shawn M. Abernathy, Kelly R. Brown \\ Department of Chemistry, Howard University, Washington DC, USA \\ Email: SAbernathy@howard.edu
}

Received 5 September 2015; accepted 21 September 2015; published 28 September 2015

Copyright (C) 2015 by authors and OALib.

This work is licensed under the Creative Commons Attribution International License (CC BY). http://creativecommons.org/licenses/by/4.0/

(c) (i) Open Access

\section{Abstract}

The objective of this investigation was to develop a vapor pressure (VP) acquisition system and methodology for performing temperature-dependent VP measurements and predicting the enthalpy of vaporization $\left(\Delta H_{\mathrm{vap}}\right)$ of volatile organic compounds, i.e. VOCs. High quality VP data were acquired for acetone, ethanol, and toluene. VP data were also obtained for water, which served as the system calibration standard. The empirical VP data were in excellent agreement with its reference data confirming the reliability/performance of the system and methodology. The predicted values of $\Delta H_{\text {vap }}$ for water (43.3 kJ/mol, $\left.1.0 \%\right)$, acetone $(31.4 \mathrm{~kJ} / \mathrm{mol} ; 3.4 \%)$, ethanol $(42.0 \mathrm{~kJ} / \mathrm{mol}$; $1.0 \%)$ and toluene (35.3 $\mathrm{kJ} / \mathrm{mol} ; 5.4 \%)$ were in excellent agreement with the literature. The computed values of $\Delta S_{\text {vap }}$ for water $(116.0 \mathrm{~J} / \mathrm{mol} \cdot \mathrm{K})$, acetone $(95.2 \mathrm{~J} / \mathrm{mol} \cdot \mathrm{K})$, ethanol $(119.5 \mathrm{~J} / \mathrm{mol} \cdot \mathrm{K})$ and toluene $(92.0 \mathrm{~J} / \mathrm{mol} \cdot \mathrm{K})$ compared also favorably to the literature.

\section{Keywords}

Vapor Pressure (VP), Enthalpy of Vaporization, Entropy of Vaporization, Volatile Organic Compounds (VOCs), Predict

Subject Areas: Physical Chemistry

\section{Introduction}

Vapor pressure (VP) is a significant physical property of liquid volatile organic compounds (VOCs). These types of compounds have low boiling points, readily evaporate, and are highly flammable. Solvents, paint thinners, hydraulic fluids, dry cleaning chemical, gasoline, and aviation fuel(s) are a few examples of VOCs and 
VOCs mixtures. These VOCs are common place in the everyday life of consumers. A substantial number of VOCs are human and environmental toxins. Most VOCs have their origin from oil refineries since they are typically produced by the fractional distillation of crude oil [1]. The VP of a VOC is temperature-dependent, and numerous thermodynamic parameters can be readily computed using VP data. For example, the enthalpy of vaporization $\left(\Delta H_{\text {vap }}\right)$ and entropy of vaporization $\left(\Delta S_{\text {vap }}\right)$ are two thermodynamic entities that can be derived from VP data of VOCs. In the petroleum industry, simulations of $\Delta H_{\text {vap }}$ are used extensively for carrying out assessment and optimization of processes [2].

Ethanol (EtOH) and toluene are two of the many VOCs that are typically in the formulation of automotive gasoline. Commercial gasoline is refined product of crude oil consisting of a mixture of hydrocarbons, additives, and blending agents. EtOH is the most commonly employed bio-fuel in gasoline formulations, where it is an oxygenated additive that is used to improve the performance (anti-knocking) and reduce automotive emissions [3]. Toluene is one of the numerous aromatic compounds found in gasoline formulations. The typical aromatics found in gasoline blends are benzene, toluene, ethylbenzene, and xylenes (BTEX). Their total composition (BTEX) in a gasoline blends can range from $20 \%-50 \% \mathrm{v} / \mathrm{v}$ (volume/volume). Toluene is also a well known gasoline surrogate fuel (TRF = toluene reference fuel) [4]. In a review article by Pitz et al. [5] it recommended that three of constituents in any gasoline and diesel fuel surrogate should be n-heptane, iso-octane, and toluene [6].

VP is a fundamental physicochemical property that is widely used for ascertaining the level of volatile compounds emitted into the atmosphere from diesel fuel and automotive gasoline. The Reid vapor pressure (RVP) is the method of choice for determining the volatility of commercial gasoline in the refinery industry. It is determined at $37.8^{\circ} \mathrm{C}\left(100^{\circ} \mathrm{F} / 311.0 \mathrm{~K}\right)$ and a vapor to liquid ratio of $4: 1$. The protocol for performing RVP measurements is described by ASTM D-323 (American Standard for Testing Materials). However, the true vapor pressure (TVP) is probably more conducive for determining the concentration of combustion contaminants emitted into the atmosphere. TVP is the pressure exerted by a vapor in equilibrium with its liquid phase at a specific temperature. An alarming number of derailment accidents in 2014 during the transport of crude oil compelled the United States Department of Transportation (USDOT) and Trans Canada, USDOT counter-part agency, to issue an emergency testing order to ensure safe transportation of crude oil via rail. To meet this safety order, the true VP of crude oil and gasoline would be measured at a series of temperature and not just one temperature as prescribed by the RVP [7].

The objective of this investigation was to acquire high quality VP data using an enhanced VP acquisition system at a series of temperatures for three VOCs. The targeted VOCs were acetone, EtOH, and toluene. Acetone is a common solvent that is typically found in homes as fingernail polish remover. EtOH is a gasoline octanebooster, and toluene is a surrogate fuel. The VP of distilled water was also measured as a reference/calibration standard. Measurements were performed on these model VOCs in order to examine the viability, efficiency, and optimize the performance of our enhanced VP acquisition system. The volatility of a fuel in the petroleum industry is typically described either by a distillation curve, the RVP, and enthalpy of vaporization $\left(\Delta H_{\text {vap }}\right)$. It can also be characterized by all three, where $\Delta H_{\text {vap }}$ is characteristically derived from VP. Accordingly, the acquired data were also used to predict the enthalpy of vaporization $\left(\Delta H_{\text {vap }}\right)$ and compute the entropy of vaporization $\left(\Delta S_{\text {vap }}\right)$ of these VOCs. The results of this investigation are presented in this paper.

\section{Experimental}

\subsection{Materials}

The VOCs acetone, anhydrous ethanol (EtOH), and toluene were purchased from the Aldrich Chemical Company. These chemicals were used without purification and handled using proper safety procedures as stipulated in their Material Safety Data Sheet.

\subsection{Methodology}

Temperature-dependent VP data was acquired for distilled water, acetone, EtOH, and toluene using our in-house VP acquisition system. The apparatus and protocol used by the system is based off of the "Boiling-Point Method", which is well recognized in the literature [8]. In this procedure, liquid vapors are in equilibrium with its boiling liquid at a specific externally applied pressure. A $250 \mathrm{ml}$ round-bottom (rb) flask served as the liquid reservoir and contained $100 \mathrm{ml}$ of VOC during data acquisition. A portable direct-drive vacuum pump (Welch 
GEM model) was utilized as the external vacuum source. A digital vacuum regulator (Model 200 DVR, manufactured by J-KEM Scientific) was coupled to the vacuum pump and employed to accurately control the pressure $( \pm 0.1$ torr $)$ above the liquids.

The VOCs and water were heated using a Büchi model B-490 water bath interfaced with a J-KEM Scientific digital temperature controller (DTC). The liquid reservoir was submerged in the water bath to equilibrate the sample at a predetermined set temperature in which the VP would be measured. For temperatures above $100^{\circ} \mathrm{C}$, a heating mantle was used in lieu of the water bath. The temperature of the sample was measured by placing the thermocouple sensor component $\left( \pm 0.1^{\circ} \mathrm{C}\right)$ of the DTC in the center of the liquid(s).

The DVR and DTC were interfaced to a desktop PC via a USB cable. Temperature (T) and pressure (P) datawere logged real-time, every 20 seconds, into an excel spreadsheet. The resultant VP data can be readily evaluated in the excel spreadsheet or exported for processing using a different software package. At least nine VP measurements were acquired at each specified temperature to ensure reproducibility of the data. This would correspond to a total acquisition time of at least three minutes. A schematic diagram of the experiment enhanced VP acquisition system is illustrated in Figure 1.

\section{Result and Discussion}

Our in-housed built VP acquisition system was used to amass VP data for distilled water, acetone, ethanol, and toluene in this investigation. The variation of VP with temperature is well described by the phase equilibrium Clapeyron equation that is expressed by Equation (1):

$$
\frac{\mathrm{d} P}{\mathrm{~d} T}=\frac{\Delta H_{\mathrm{vap}} P}{R T^{2}}
$$

The variables $P$ and $T$ in Equation (1) are the vapor pressure and the absolute temperature (K $=$ Kelvin) respectively. The enthalpy of vaporization is denoted by $\Delta H_{\text {vap }}$ and $R$ is the gas constant $(8.314 \mathrm{~J} / \mathrm{mol} \cdot \mathrm{K})$. The

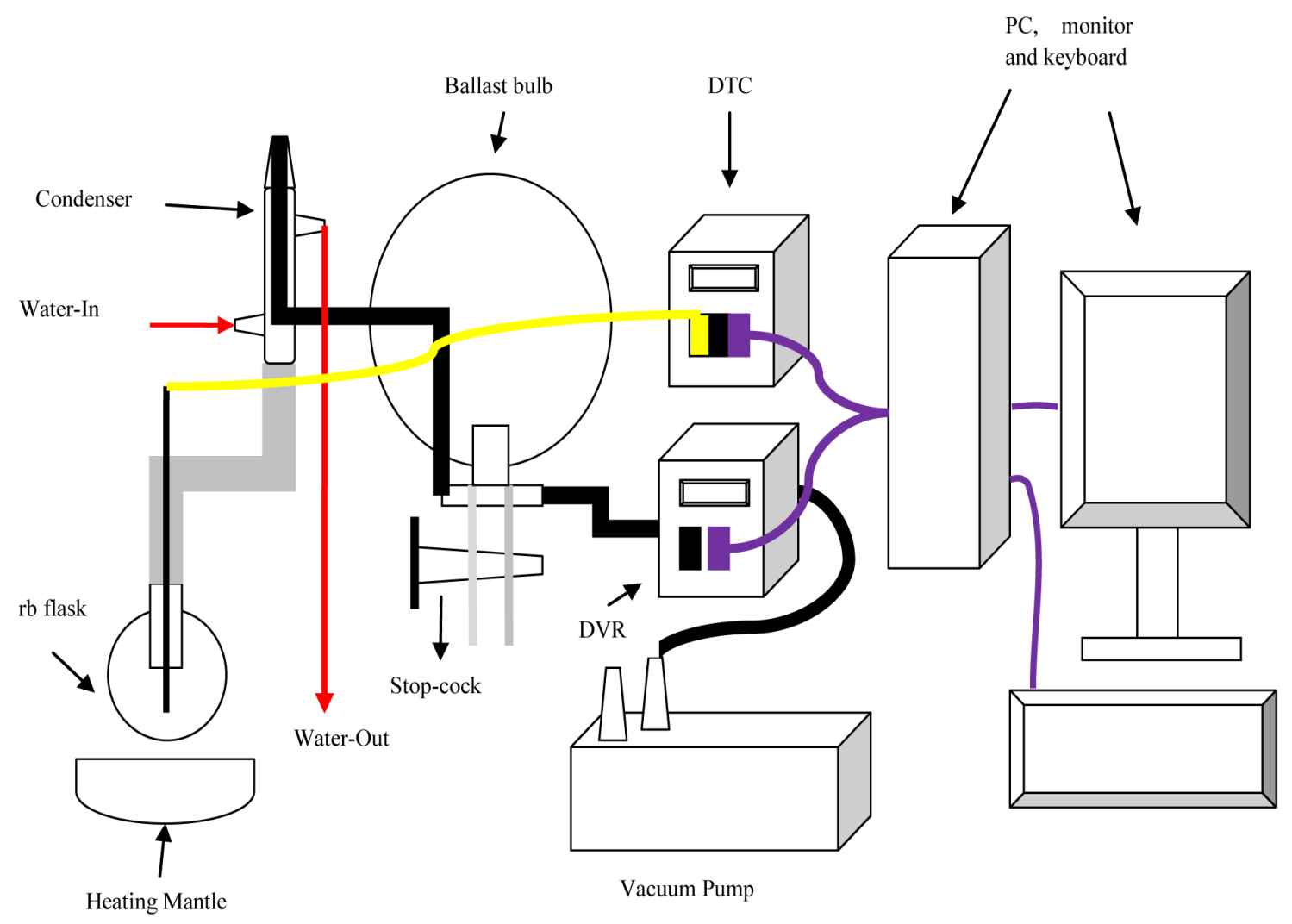

Figure 1. Schematic diagram of the enhanced vapor pressure acquisition system used. 
integration of the Clapeyron equation yields the Clausius-Clapeyron, which is a linear equation represented by Equation (2):

$$
\ln P=-\frac{\Delta H_{\text {vap }}}{R T}+C
$$

The enthalpy of vaporization can be predicted from Equation (2) from a plot of the natural logarithm of pressure $(\ln P)$ versus the reciprocal absolute temperature $(1 / K)$. A straight line is generated with a slope equal to $-\Delta H_{\text {vap }} / R$ and an intercept denoted by the constant $C$ [9]. This is the method utilized to compute $\Delta H_{\text {vap }}$ in this study. A plot of the empirical VP data of water as the $\ln P$ vs. $1 / T(K)$ is shown in Figure 2.

A linear least square regression fit was applied to each set of VP data in order to predict $\Delta H_{\text {vap }}$ and calculate $\Delta S_{\text {vap }}$ for each VOC. Using the water VP data as an example, a linear least square regression fit of the data results in an equation for the line of best fit equal to $y=20.657-5215.1 x$. This can be translated to the form of Equation (2) as:

$$
\ln P=\frac{-5215.1}{T}+C
$$

A value of $43.4 \mathrm{~kJ} \cdot \mathrm{mol}^{-1}$ was predicted as the enthalpy of vaporization $\left(\Delta H_{\text {vap }}\right)$ of water after multiplying the slope of the resultant line fit by $8.314 \mathrm{~J} / \mathrm{mol} \cdot \mathrm{K}$, i.e. the gas constant. The predicted value for water deviated by only $1.0 \%$ from the literature value for water [10] [11]. The correlation coefficient $(R)$ for the line fit was 0.99989 , which indicate an excellent fit to the data since the value is close to 1 . The entropy of vaporization is computed by dividing $\Delta H_{\text {vap }}$ of water by it normal boiling point temperature in Kelvin $\left(100.0^{\circ} \mathrm{C}, 373.2 \mathrm{~K}\right)$; this corresponds to a $\Delta S_{\text {vap }}$ of $116.3 \mathrm{~J} / \mathrm{mol} \cdot \mathrm{K}$ for water.

The predicted values of $\Delta H_{\text {vap }}$ for acetone, EtOH and toluene were $31.4 \mathrm{~kJ} / \mathrm{mol}, 42.0 \mathrm{~kJ} / \mathrm{mol}$, and $35.3 \mathrm{~kJ} / \mathrm{mol}$ respectively using the line of best fit through the empirical VP data. The plot of the $\ln P$ versus $1 / T(\mathrm{~K})$ for acetone, EtOH, and toluene are shown in Figures 3-5 respectively. The $\Delta H_{\text {vap }}$ of acetone, EtOH and toluene deviated by $3.4 \%, 1.0 \%$ and $5.4 \%$, which compare favorable with the literature values [11]. The computed values for $\Delta S_{\text {vap }}$ of acetone, EtOH and toluene were $95.2 \mathrm{~J} / \mathrm{mol}, 119.5 \mathrm{~J} / \mathrm{mol} \cdot \mathrm{K}$ and $35.3 \mathrm{~J} / \mathrm{mol} \cdot \mathrm{K}$ respectively, where $\Delta S_{\text {vap }}$ of each VOCs is equal to $\Delta H_{\text {vap }} / \mathrm{T}_{\mathrm{b}}$, Kelvin.

Table 1 is a synopsis of the VP data amassed for water, acetone, EtOH, and toluene. It contains the predicted value of $\left(\Delta H_{\text {vap }}\right)$, the computed values of $\left(\Delta S_{\text {vap }}\right)$, the slope of the line fit, and the correlation coefficient. From Table 1, it is readily apparent that the experimental data parallels the literature data, which are shown in parentheses. The results of this investigation clearly demonstrate the enhanced viability and efficiency of our enhanced VP acquisition system for amassing VP data.

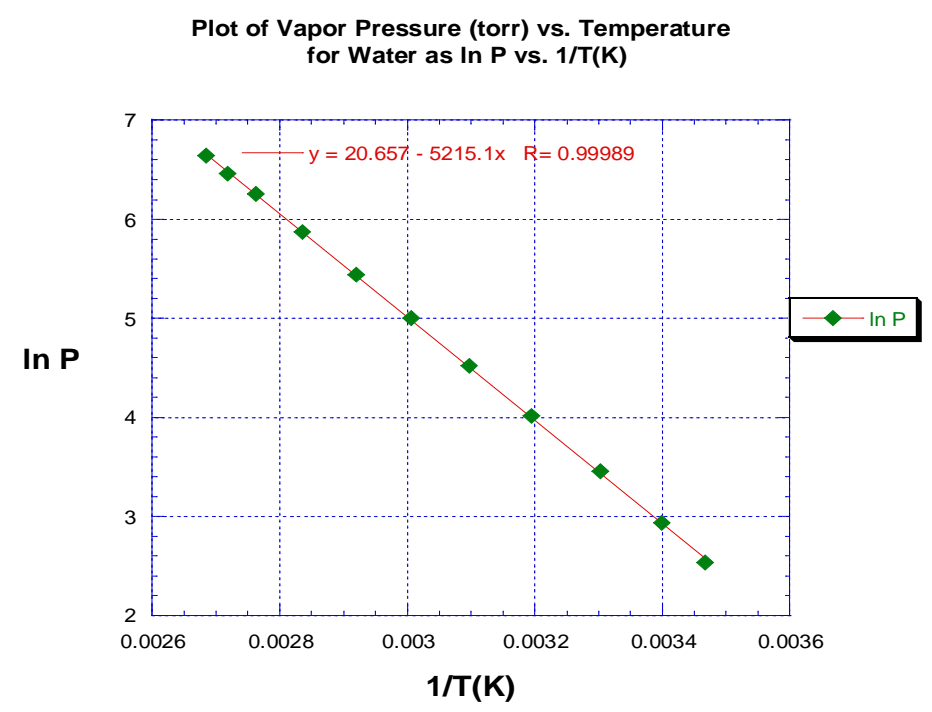

Figure 2. Plot and linear least squares fit of the VP of water. 


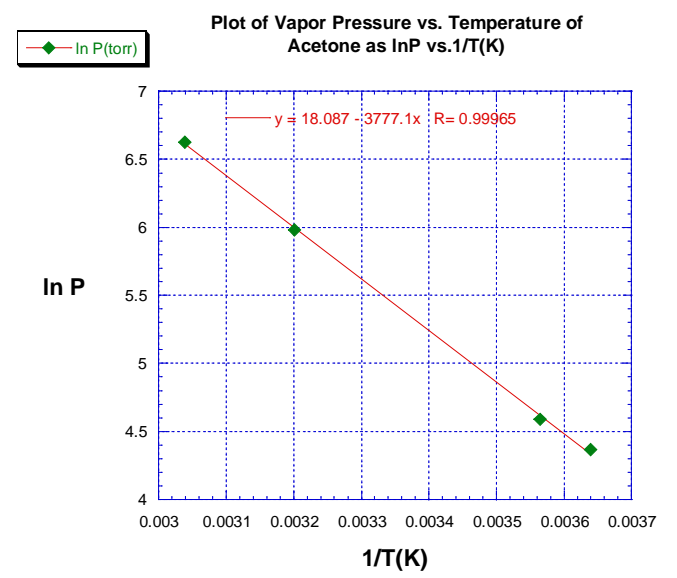

Figure 3. Plot and linear least squares fit of the VP of acetone.

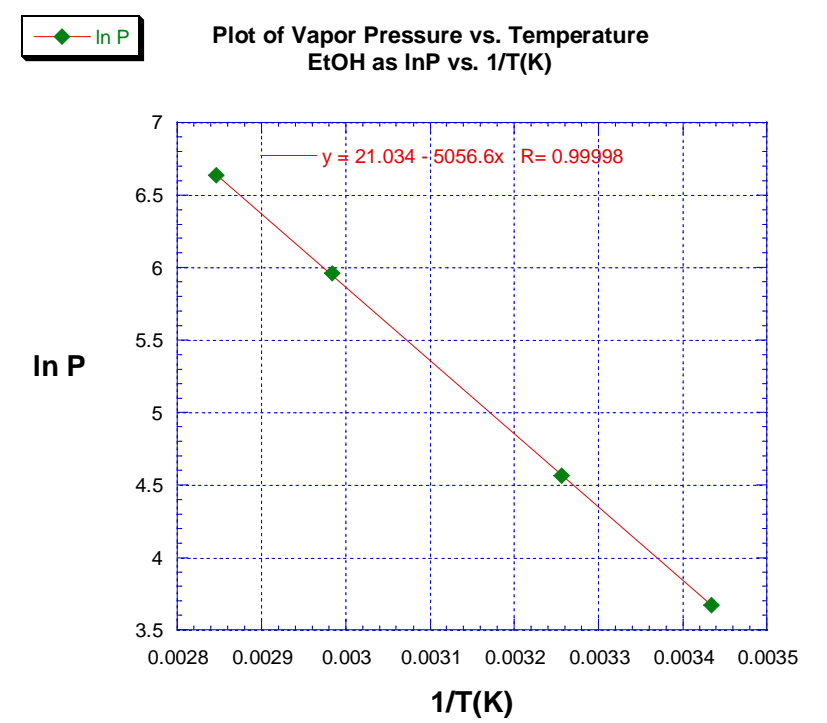

Figure 4. Plot and linear least squares fit of the VP of EtOH.

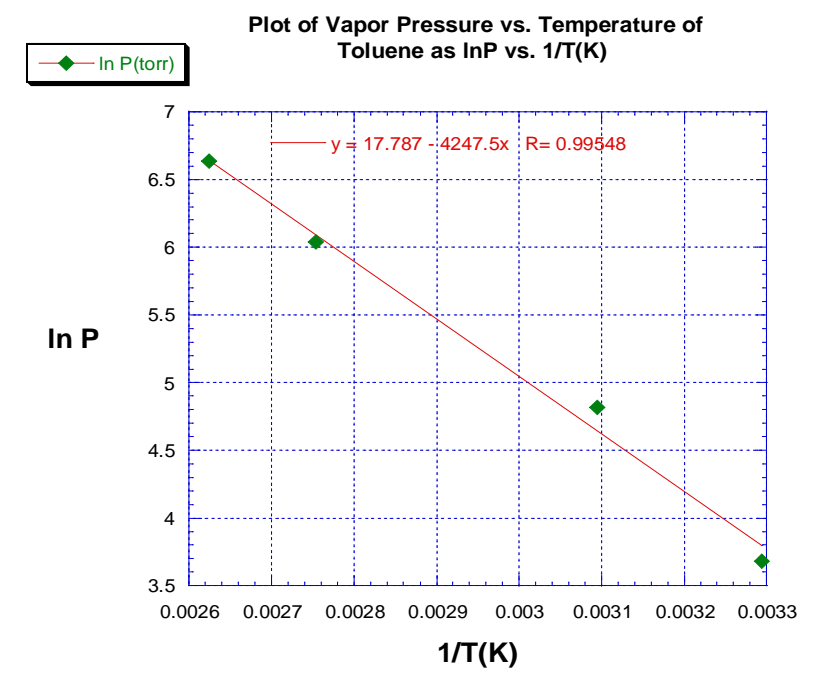

Figure 5. Plot and linear least squares fit of the VP of toluene. 
Table 1. Summary of predicted and computed results from VP data amassed from the enhanced VP acquisition system.

\begin{tabular}{|c|c|c|c|}
\hline Liquids & Temp. ${ }^{\circ} \mathrm{C}$ (Lit. value) & $\begin{array}{l}\text { Pressure Torr. } \\
\text { (Lit. value) }\end{array}$ & $\begin{array}{l}\Delta \mathrm{H}_{\mathrm{Vap}} \& \Delta \mathrm{S}_{\mathrm{Vap}} \\
\text { From Line Fit }\end{array}$ \\
\hline \multirow[t]{11}{*}{ Water } & 15.2 & 12.5 (12.953) & $43.3 \mathrm{~kJ} / \mathrm{mol}$ \\
\hline & 21.0 & $18.8(18.650)$ & $116.0 \mathrm{~J} / \mathrm{mol} \cdot \mathrm{K}$ \\
\hline & 29.6 & 31.6 (34.864) & Slope $=-5215.1$ \\
\hline & 39.8 & $55.0(54.156)$ & $\mathrm{R}=0.99989$ \\
\hline & 49.7 & $91.5(91.14)$ & \\
\hline & 59.5 & $148.1(146.0)$ & \\
\hline & 69.3 & 230.9 (226.7) & \\
\hline & 79.3 & 351.6 (345.4) & \\
\hline & 88.9 & $520.1(504.2)$ & \\
\hline & 94.7 & $636.8(627.0)$ & \\
\hline & 99.4 & 766.1 (743.85) & \\
\hline \multirow[t]{4}{*}{ Acetone } & 1.5 & 79.1 & $31.4 \mathrm{~kJ} / \mathrm{mol}$ \\
\hline & $7.3(7.7)$ & $98.6(100)$ & $95.2 \mathrm{~J} / \mathrm{mol} \cdot \mathrm{K}$ \\
\hline & $39.2(39.5)$ & $396.0(400)$ & Slope $=-3777.1$ \\
\hline & $55.9(56.5)$ & $753(760)$ & $\mathrm{R}=0.99965$ \\
\hline \multirow[t]{4}{*}{$\mathrm{EtOH}$} & $18.0(19.0)$ & $39.2(40)$ & $42.0 \mathrm{~kJ} / \mathrm{mol}$ \\
\hline & 33.9 (34.9) & $96.2(100)$ & $119.5 \mathrm{~J} / \mathrm{mol} \cdot \mathrm{K}$ \\
\hline & $62.0(63.5)$ & $387.0(400)$ & Slope $=-5056.6$ \\
\hline & $78.1(78.4)$ & $761.6(760)$ & $\mathrm{R}=0.99998$ \\
\hline \multirow[t]{4}{*}{ Toluene } & 30.4 (31.8) & $39.8(40)$ & $35.3 \mathrm{~kJ} / \mathrm{mol}$ \\
\hline & $49.9(51.9)$ & $123.5(100)$ & $92.0 \mathrm{~J} / \mathrm{mol} \cdot \mathrm{K}$ \\
\hline & 89.9 (89.5) & $418.2(400)$ & Slope $=-4247.5$ \\
\hline & $107.9(110.6)$ & $759.7(760)$ & $\mathrm{R}=0.99548$ \\
\hline
\end{tabular}

\section{Conclusion}

Vapor pressure (VP) data were acquired for water, acetone, ethanol, and toluene using the in-house built enhanced VP acquisition system. The empirical data were used to predict the enthalpy of vaporization $\left(\Delta H_{\mathrm{vap}}\right)$ from the Clausius-Clapeyron equation and compute the entropy of vaporization $\left(\Delta S_{\text {vap }}\right)$ for water and the VOCs. The acquire VP was in excellent agreement with the literature data for the liquids. The predicted values of $\Delta H_{\text {vap }}$ and the computed values for $\Delta S_{\text {vap }}$ compared quite favorable to the literature values. These results confirm the performance/reliability and efficiency of the VP acquisition system and its experimental protocol. Future studies will entail the use of the system to examine viscose oils such as mineral oil and motor oil as well as mixtures of VOCs.

\section{Acknowledgements}

The authors acknowledge Howard University, Dr. Clarence Lee (Executive Director) of the Howard University LS-AMP (Louis Stokes Alliance for Minority Participation) Program, Marquia Whitlock (LS-AMP Program), Monique Yvette McClung (LS-AMP Program), NSF (Grant Number HRD-1000286), and NIH-NIGMS (Grant Number T34GM105660).

\section{References}

[1] Girard, J.E. (2005) Principles of Environmental Chemistry. Jones and Bartlett Publishers, MA. 
[2] Gopinathan, N. and Sarai, D.N. (2001) Predict Heat of Vaporization of Crudes and Pure Components Revised II. Fluid Phase Equilibria, 179, 277-284. http://dx.doi.org/10.1016/S0378-3812(00)00501-X

[3] Andersen, V.F., Andersen, J.E., Wallington, T.J., Mueller, S.A. and Nielsen, O.J. (2010) Vapor Pressure of AlcoholGasoline Blends. Energy Fuels, 24, 3647-3654. http://dx.doi.org/10.1021/ef100254w

[4] Andrae, J.C.G., Brinck, T. and Kalghatgi, T.B. (2008) HCCI Experiment with Toluene Reference Fuels Modeled by a Semidetailed Chemical Kinetic Model. Combustion and Flame, 155, 696-712. http://dx.doi.org/10.1016/j.combustflame.2008.05.010

[5] Pitz, W.J., Cernansky, N.P., Dryer, F.L., Egolfopoulos, F.N., Farrell, J.T., Friend, D.G. and Pitsch, H. (2007) Development of an Experimental Database and Kinetic Models for Surrogate Diesel Fuels. SAE Technical Paper, 2007-010175. http://papers.sae.org./2007-01-0175/

[6] Andrae, J.C.G. and Head, R.A. (2009) HCCI Experiment with Gasoline Surrogate Fuel Modeled by a Semidetailed Chemical Kinetic Model. Combustion and Flame, 156, 842-851. http://dx.doi.org/10.1016/j.combustflame.2008.10.002

[7] Pichter, H. and Lutz, J. (2014) Why Crude Oil Vapor Pressure Should Be Tested Prior to Rail Transport. Advances in Petroleum Exploration and Development, 7, 58-61.

[8] Garland, C.W., Nibler, J.W. and Shoemaker, D.P. (2009) Experiments in Physical Chemistry. 8th Edition, McGrawHill, New York.

[9] Atkins, P. and Paula, J. (2010) Physical Chemistry. 9th Edition, W. H. Freeman Co., New York.

[10] Weast, R.C., Astle, M.J. and Beyer, W.H. (1984) CRC Handbook of Chemistry and Physics. CRC Press, Boca Raton, 199-214.

[11] http://webbook.nist.gov/chemistry/form-ser.html 